\title{
Towards easier and more functional semantics for geometrical tolerancing
}

\author{
E. Pairel, M. Giordano, S. Samper \\ LMécA/CESALP (Laboratoire de Mécanique Appliquée) \\ Ecole Supérieure d'Ingénieurs d'Annecy - Université de Savoie \\ BP 806, 74016 ANNECY Cedex - FRANCE \\ pairel@esia.univ-savoie.fr
}

\begin{abstract}
Geometrical defects of a manufactured feature are limited by form, size and location tolerances. The standardized semantics of those tolerances, i.e. their interpretation on the manufactured feature, presents a lot of drawbacks. We propose new semantics, based on the fitting of a unique theoretical feature on the manufactured feature. The fitting criterion we propose, named minimum volume criterion, leads to the actual mating envelope of the manufactured feature. For some functional requirements the tolerancing shown to become simpler and more functional.
\end{abstract}

Keywords: tolerancing, semantics, fitting criterion, minimum volume criterion

\section{THE THREE DEFECTS OF A FEATURE: FORM, SIZE, LOCATION}

The tolerancing principle consists in considering that the actual feature presents three kinds of defect: form, size and location defects.

Consequently, we find three types of tolerances for each feature (figure 1.a).

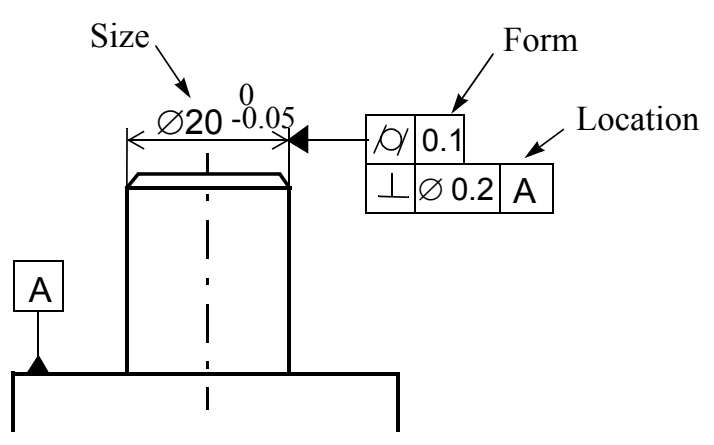

(a)

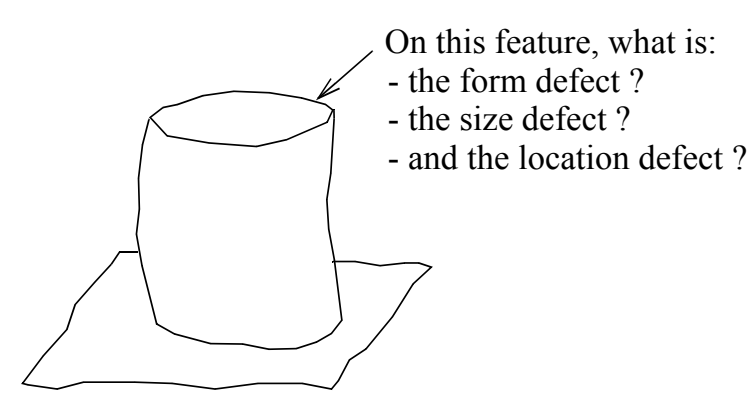

(b)

Figure 1; The three tolerances of a feature

Note: the word «location» will be used to refer indifferently to location or orientation tolerance. 
Let us note that this division in three defects is arbitrary because the actual feature has a shape on which form, size and location defects are not intrinsically defined (figure 1.b). So defining those different defects regardless of the actual feature is necessary.

The main study on this problem is due to A.A.G. Requicha [Requicha 1983]. But the semantics that he has proposed is not really functional and does not allow to define the values of form, size and location defects of an manufactured feature.

The other main approach consists in defining these three defects with a theoretical feature fitted to the manufactured feature [Hillyard 1978] [Wirtz 1989].

Let us note that the standardized semantics noes not correspond to any of these two approaches.

\section{PROPOSED SEMANTICS}

We propose to define the form, size and location defects of the actual feature by comparing it to a unique theoretical feature fitted to the actual feature :

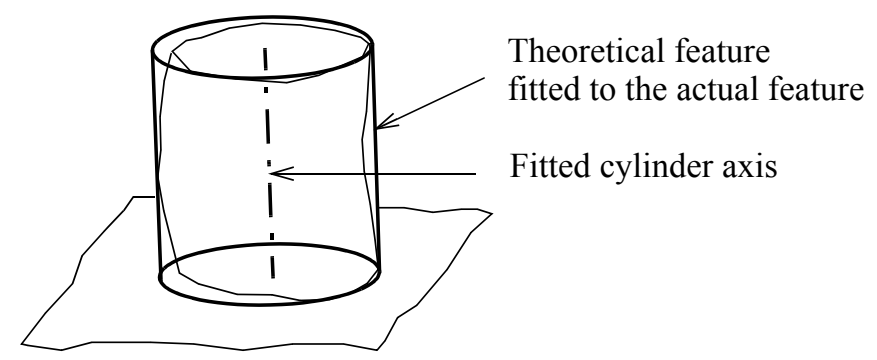

Figure 2; Theoretical feature fitted to define the defects of a surface

The fitting criterion that we choose, presented in section 4, leads to obtain the actual mating envelope of the manufactured feature. For a shaft, it is the smallest theoretical cylinder fitting the outside of the shaft. Nevertheless, the designer should be able to choose another criterion.

\subsection{Size tolerance semantics}

The size tolerance concerns the size of the fitted feature. Its value has to be between the minimum and the maximum sizes authorized by the tolerance.

The size defect is the deviation between the size of the actual matting envelope and the nominal size defined by the designer.

\section{Case of size tolerance applied to angle :}

The standards allow the size tolerancing of the angle between two planes. However the standardized interpretation of such a tolerance is ambiguous because of its incomplete definition. With the proposed semantics, there is no ambiguity: the angle tolerance concerns the angle between the two planes fitted to the manufactured features. 
The 6th CIRP International Seminar on Computer-Aided Tolerancing, March 1999, University of Twente, Enschede, The Netherlands

\subsection{Form tolerance semantics}

The form defect is the maximum distance between the theoretical feature and the actual feature along a perpendicular direction to the theoretical feature. It has to be inferior to the form tolerance. This semantics is very close to standardized semantics.

\subsection{Location tolerance semantics}

The location tolerance concerns the fitted feature axis along the nominal height. This axis, limited by two extreme points, has to be within the tolerance zone defined by the location tolerance:

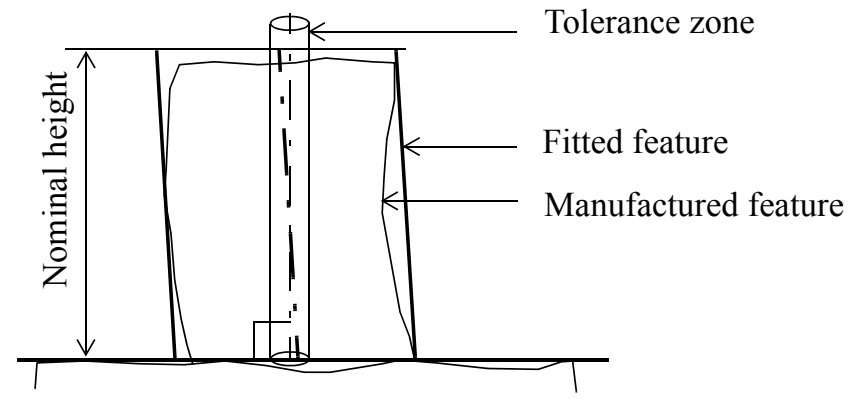

Figure 3; New semantics for a location/orientation tolerance

The location defect can be defined as the size of the minimum tolerance zone.

\section{STANDARDIZED SEMANTICS}

\subsection{Standardized interpretation of the size tolerance}

International standards [ISO 8015] indicate that the size tolerance only limits the «actual local sizes» of the actual feature :

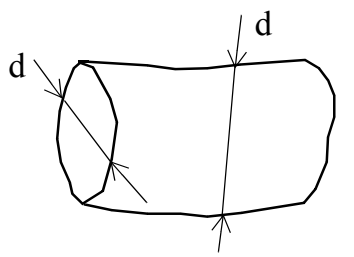

Figure 4; Standardized semantics of a size tolerance

This semantics is based on the shop floor practices using micrometers and callipers which enable to measure the distance between two points.

Different studies were conducted to give a definition of the actual local size [Srinivasan 93]. The proposed definitions are complex and lead to more difficulties in metrology.

Whatever its definition, the actual local size concept involves problems for the geometrical specification and verification of products because local sizes do not always exist on parts. 
For instance, in many cases, the tolerances cannot always be verified on every point of the feature:

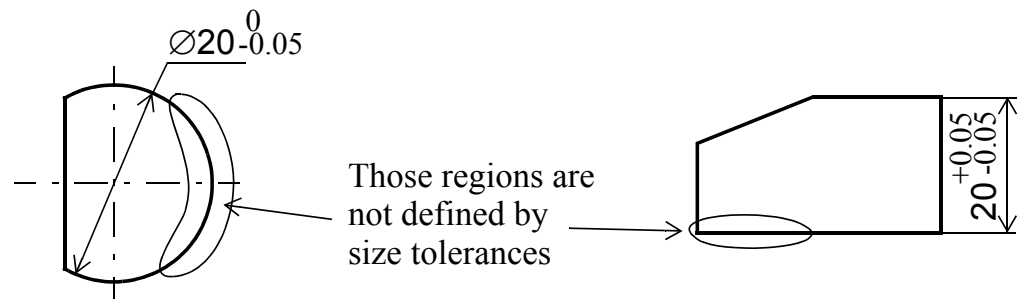

Figure 5; Features incompletely defined by the size tolerance

A lot of specifications are irrelevant with the standardized interpretation :
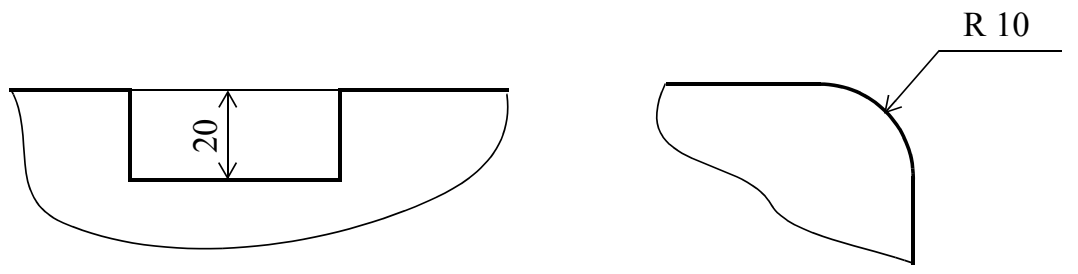

Figure 6; Irrelevant specifications with the standardized semantics

With the proposed semantics:

With the proposed semantics, each one of the above specifications is relevant.

For cylindrical features, it is always possible to fit theoretical cylinder on them even if they are not complete.

For a size tolerance between two planes, the theoretical entity to be fitted is composed of two parallel planes with a variable distance :
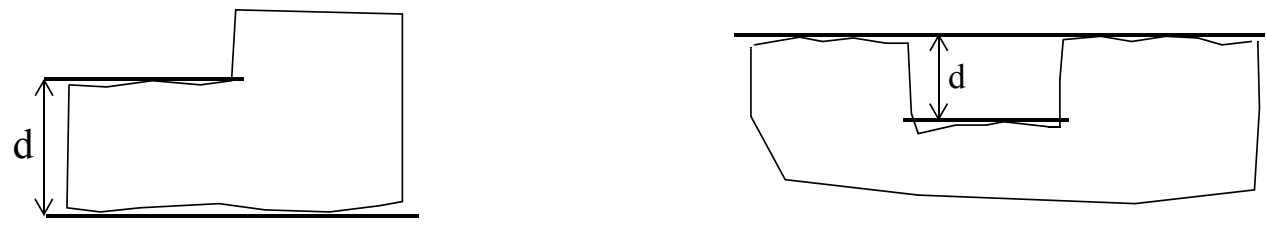

Figure 7; Two parallel plane entity

Furthermore, interpretation in terms of actual local sizes has not any real functional justification. So, in our opinion, this concept should be abandoned for a interpretation based on feature fitting.

\subsection{Standardized interpretation of a form tolerance}

The manufactured feature has to be between two theoretical features obtained by offsetting of the nominal feature to the exterior and interior sides.

This condition can be verified by fitting the exterior offsetted feature on the actual feature, with the minimum form defect criterion. So, the only difference between the standardized interpretation and the proposed interpretation is the fitting criterion. 
The 6th CIRP International Seminar on Computer-Aided Tolerancing, March 1999, University of Twente, Enschede, The Netherlands

\subsection{Standardized interpretation of a location tolerance}

According to the standardized interpretation, a location tolerance concerns an «actual» feature of the part.

For the cylinder, the concerned feature is the «actual axis» of the feature :

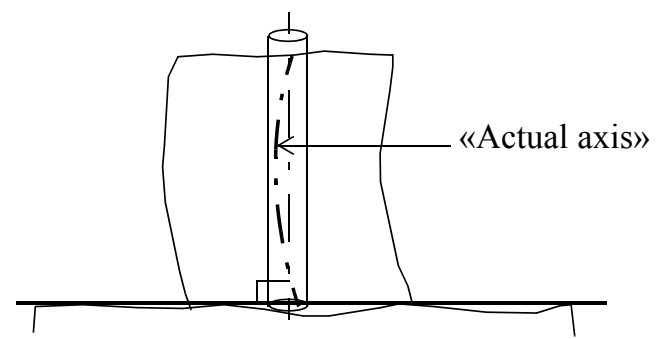

Figure 8; Standardized interpretation of a location tolerance

But the «actual axis» is a virtual feature on the manufactured part. So it is necessary to define how this feature is built from the actual feature. In our knowledge, standards on tolerancing do not give this definition. This gap leads to several metrology practices:

- The first method consists in building approximate centers of some sections of the shaft.

- The second method consists in verifying the location of a perfect feature fitted to the manufactured feature.

Although this method is not consistent with standardized interpretation, it is more and more commonly used because of the growing use of CMM.

Besides, it is generally more adapted to the designer's intention.

The semantics proposed here corresponds to this verification method.

\section{Projected tolerance zone:}

A location tolerance with a projected tolerance zone enables to specify that the continuation of the actual feature has to respect the location tolerance (figure 9.a).
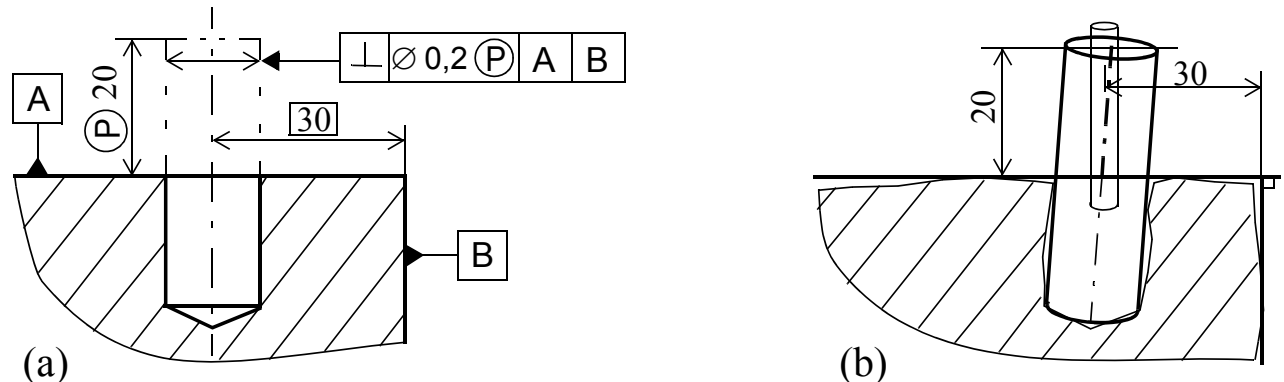

Figure 9; Projected tolerance zone specification and interpretation

In this case, it is impossible to consider the «actual axis» of the hole. The axis concerned by the specification is necessarily the axis of a theoretical feature fitted to the hole (figure 9.b). So, the standardized interpretation is not coherent with this kind of specification. On the other hand, the proposed semantics is relevant in this case. 
The 6th CIRP International Seminar on Computer-Aided Tolerancing, March 1999, University of Twente, Enschede, The Netherlands

\section{DEFINITION OF THE FITTED FEATURE - MINIMUM VOLUME CRITERION}

The fitted feature has to represent the manufactured feature as functionally as possible. In our opinion, it has to be the actual mating envelope of the manufactured feature so as to allow to control the mating requirement directly.

Thus an original and general fitting criterion is proposed, named the minimum volume criterion.

It consists in defining the theoretical feature, outside of the material, which minimizes the volume of the space between the theoretical and the manufactured features. Figures below illustrate fittings of a plane and of a cylinder according to this criterion.

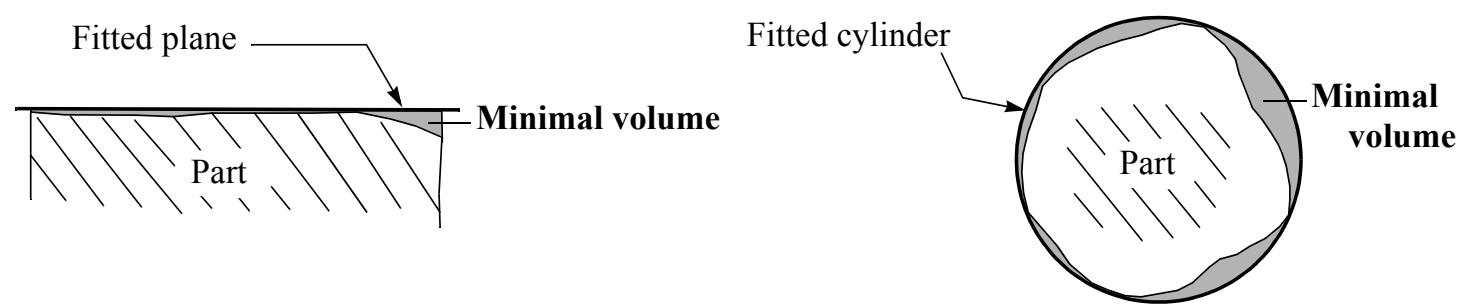

Figure 10; Fittings according to the minimum volume criterion

In the case of the cylinder, the minimum volume criterion leads to the smallest size theoretical cylinder fitting the outside of the shaft.

In some cases, the minimum volume criterion can give several solutions, i.e. several theoretical features minimizing the volume. The chosen feature will be the one which also minimizes the maximum distance between the theoretical and the manufactured features.

The theoretical features are better fitted to actual features with the minimum volume criterion than by other criteria. In particular, the minimum form defect criterion, which consists in minimizing the maximum distance between the theoretical feature and the actual feature, can leads to obtain fitted features more distant to actual features. The actual features in the above figure are in this case:
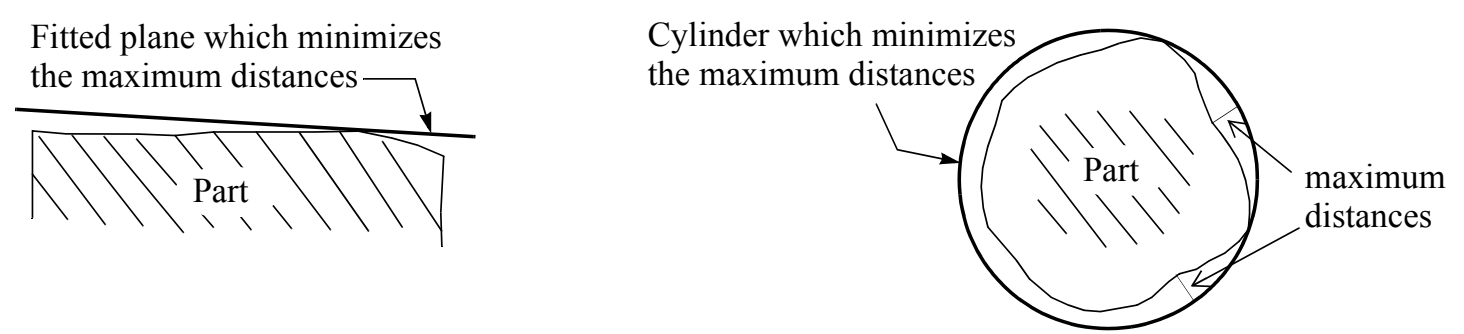

Figure 11; Exterior theoretical features obtained by the minimum form defect criterion

Another advantage of the minimum volume criterion is that it formalizes and generalizes standardized fittings of datums to actual features. 
Let us note that, with the proposed semantics, when an actual feature is a first datum feature of the part, the same theoretical fitted feature defines the datum and the geometrical defects of the actual feature.

The minimum volume criterion can be computerized [Pairel 1995]. Nevertheless, even if the least square criterion is used, the verifications done now on CMM are closer to the proposed semantics than to the standardized semantics.

\section{SOME CASES OF FUNCTIONAL TOLERANCING}

\subsection{Mating requirement}

The proposed size tolerance semantics enables to control the maximum and minimum clearances between two mated features very simply. It is more functional than the envelope requirement [ISO 8015] because it does not limit actual local sizes which are not functional for the assembly.

On the example below, the radial clearance is maximum when the actual mating envelope of the shaft has the minimum diameter allowed by the tolerance. The fact that some actual local sizes have smaller values does not increase the maximum clearance.

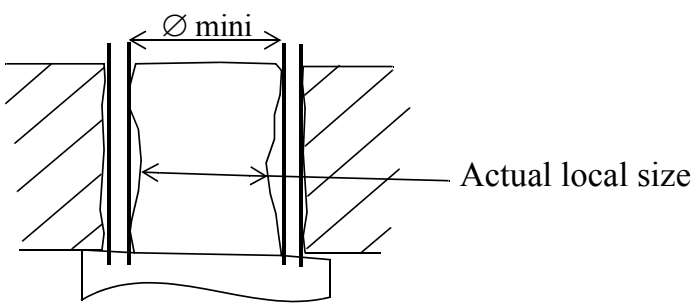

Figure 12; Actual local sizes are not functional for the assembly

With the proposed semantics, the actual local sizes result from the form defects of the actual feature. So they are limited by the form tolerance of the feature.

\subsection{Guiding precision requirement for a shaft}

For a long shaft, for example a guiding shaft for a collar, the size tolerance can be applied along part of the length of the shaft (the collar length):

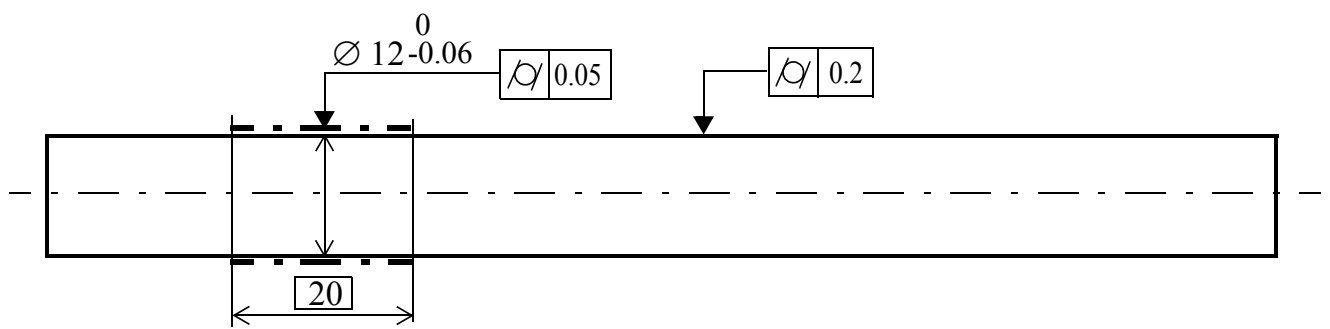

Figure 13; Proposed tolerancing for a long shaft 


\section{The 6th CIRP International Seminar on Computer-Aided Tolerancing, March 1999, University of Twente, Enschede, The Netherlands}

According to the proposed semantics, anywhere along the shaft, the diameter of the actual mating envelope, of a length of about $20 \mathrm{~mm}$, has to be between the minimum and the maximum sizes allowed by the tolerance.

The form tolerance concerns the actual mating envelope too: the distance between the shaft and any $20 \mathrm{~mm}$-long mating envelope has to be inferior to the form tolerance value.

In order to limit the straightness defect of the shaft, a cylindricity tolerance for the whole shaft is specified (figure 13). This is almost equivalent to a straightness tolerance applied to the generators of the shaft. But the semantics of this last kind of tolerance is more difficult to define.

\section{CONCLUSION}

The semantics proposed in this paper for form, size and position tolerances is greatly inspired from verifications on CMM: What is check is the size and the position of the fitted feature.

The idea to define the form, size and position defects regardless of a theoretical feature, fitted to actual feature, was already proposed by A. Wirtz [Wirtz 1989]. But the «vectorial tolerancing» that he propose, uses a very different principle of the tolerancing by tolerance zones. It is more difficult to use and less functional than the geometrical tolerancing as it is shown by S. Bialas [Bialas 1997].

The originality of the proposed semantics is the fitting criterion which leads to obtain the mating envelope of the manufactured feature. Thus, the size of the fitted feature has a functional interpretation.

The proposed semantics allows to define the form, size and position/orientation defects. Thus the geometrical verification according to this semantics enables to give the values of these defects. On the other hand, the geometrical verification according to standardized semantics can give only a binary response to the conformance question of the feature.

Furthermore, let us note that form, size and position tolerances are completely independent with the proposed semantics. Thus, the form tolerance can be larger than the position tolerance. It is not true for the standardized semantics: the position tolerance limits also the form defect of the feature. So the principle of independency between the tolerances is better respected.

Finally, the proposed semantics corresponds to the ones which are generally considered in models developed for the computer aided tolerancing systems. 
The 6th CIRP International Seminar on Computer-Aided Tolerancing, March 1999, University of Twente, Enschede, The Netherlands

\section{REFERENCES}

[Bialas 1997] Bialas S.; Humienny Z.; Kiszka K.; «Relations between ISO 1101 Geometrical Tolerances and Vectorial Tolerances - Conversion Problems»; Proceedings of 5th CIRP International Seminar on CAT; April 1997 Canada; pp. $37-48$

[Hillyard 1978] Hillyard R.C.; Braid I.C.; «Characterizing non-ideal shapes in term of dimensions and tolerances»; Computer Graphics, Vol. 12, N³, pp. 234-238

[ISO 8015:1985] ISO 8015:1985, Technical drawings - Fundamental tolerancing principle.

[Pairel 1995] E. Pairel; «Métrologie fonctionnelle par calibre virtuel sur machine à mesurer tridimensionnelle"; Thèse de Doctorat de l'Université de Savoie; 1995

[Requicha 1983] Requicha A.A.G.; «Toward a theory of geometric tolerancing», The International Journal of Robotics Research, Vol. 2, No. 4, Winter 1983.

[Srinivasan, 1993] V. Srinivasan, «Recent Effort in Mathematization of ASME/ANSI Y14.5M»; Proceedings of the 3rd CIRP Seminars on Computer Aided Tolerancing; April 1993 France; pp223-232

[Wirtz 1989] A. Wirtz, «Vectorial tolerancing»; Proceedings of the CIRP International Conference on CAD/CAM and AMT; Dec. 1989 Israel. 